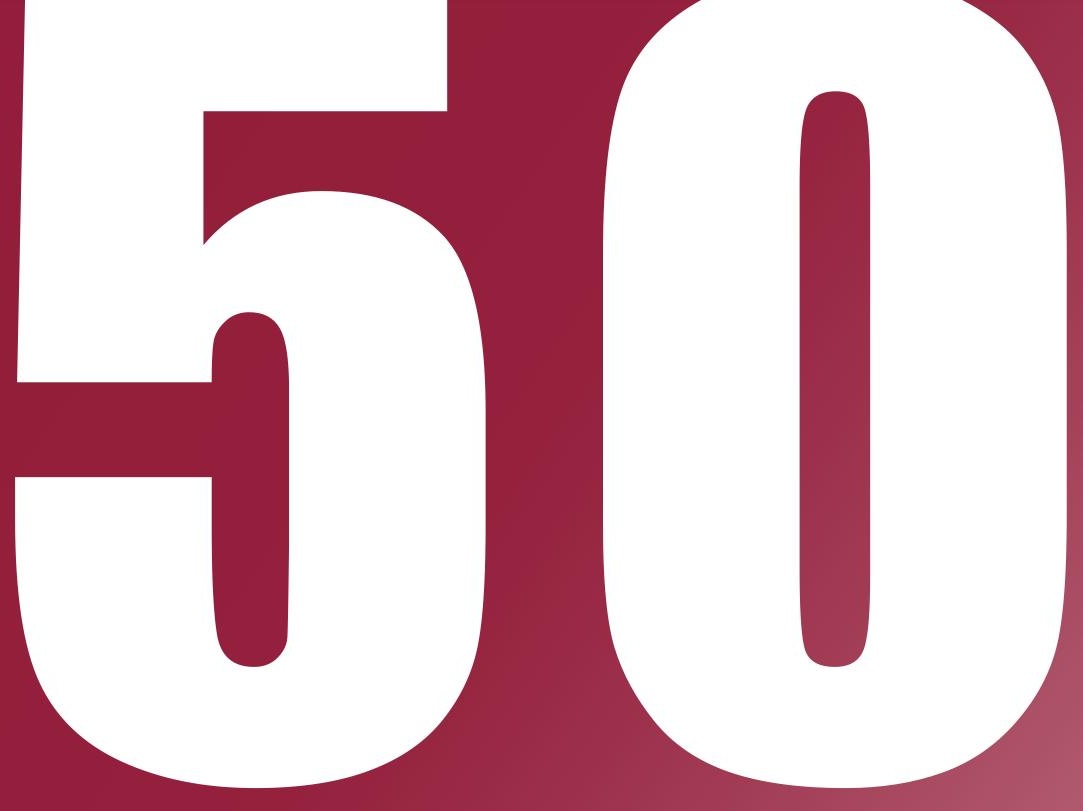

ÁMBITOS

REVISTA

INTERNACIONAL

DE

COMUNICACIÓN

$\mathbf{N}^{\circ} \mathbf{5 0}$

EDICIÓN OTOÑO

2020

ISSN: 1139-1979

E-ISSN: 1988-5733

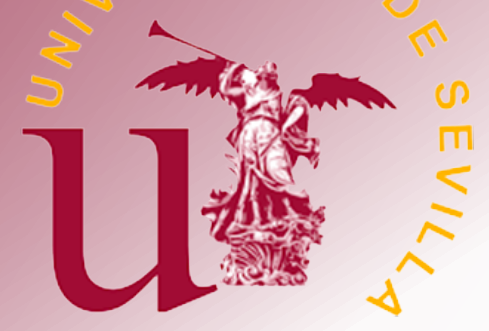




\section{INDICE}

EDITORIAL EDITORIAL

Presentación del Monográfico. Comunicación y juventud. Nuevos medios, representación, recepción y participación en contenidos de entretenimiento e información

Presentation of the monograph. Communication and youth. New media, representation, reception and participation in entertainment and information content.

Juan Francisco Gutiérrez Lozano, Francisco Javier Ruiz del Olmo

7-9

\section{MONOGRÁFICO MONOGRAPH}

El consumo audiovisual de la Generación Z. El predominio del vídeo online sobre la televisión tradicional The audiovisual consumption of Generation $Z$. The predominance of online video over traditional television

La imagen de los jóvenes en las estrategias transmedia de las series de televisión: el caso de Skam España en Instagram

The image of young people in transmedia strategies of TV series: the case of Skam España on Instagram

Sergio Jesús Villén Higueras, Francisco Javier Ruiz del Olmo

La penetración de Netflix en el público español ¿cuestiona el modelo televisivo tradicional?

Does the arrival of Netflix in the Spanish audience challenge the traditional television model?

Javier Bustos Díaz

49-61

Generación Z y consumo de información política: entre la televisión y los nuevos formatos mediáticos Generation $Z$ and consumption of political information: between television and new media formats

Ruth Gómez de Travesedo Rojas, Marta Gil Ramírez

$62-79$

Actualidad y comedia: El éxito de El Intermedio y La Resistencia entre los espectadores jóvenes

Information and comedy: The Success of El Intermedio and La Resistencia for young audiences

Inmaculada Concepción Aguilera García

80-95 
El efecto socializador de articular un espacio de comunidad virtual en el ecosistema del aula

The socializing effect of creating a virtual community space in the classroom environment

Lorea Ariadna Ruiz Gómez, Mónica Hinojosa Becerra, Francisco Javier Ruiz San Miguel

Juventude ciberativista e educação: reflexões sobre um jeito hacker de ser

Cyber activity youth and Education: reflections on a hacker way of being

Carla Azevedo de Aragão, Pietro Matheus Bompet Fontoura Alves, Karina Moreira Menezes

La risa grotesca en la obra de Bob Dylan: análisis de la Bacanal y el Festín Carnavalesco en la trilogía folk-rock eléctrica

Grotesque Laughter in Bob Dylan's Work: Analysis of The Bacchanal and the Carnival Feast in the Electric Folk-rock Trilogy

Jesús Albarrán Ligero

El papel del intertexto en el videojuego. Una partida, mil y una historias

Intertext in video games. A game, thousand and one stories

Rocío Serna-Rodrigo

145-158

\section{ÁmBitos PERSONALES PERSONAL ÁmBITOS}

El auge de Twitch: nuevas ofertas audiovisuales y cambios del consumo televisivo entre la audiencia juvenil The rise of Twitch: New audiovisual offers and the transformation of Television consumption among young audiences

\section{ARTÍCULOS ARTICLES}

La recepción de los medios de comunicación para residentes rusos en la Costa del Sol

Mass media reception by Russian residents on the Costa del Sol

Natalia Meléndez Malavé, José Carlos Pozo García

176-192

La calidad periodística en la cobertura de terremotos: Caso Ecuador

Journalistic quality in earthquake coverage: The case of Ecuador

Juan Pablo Arrobo-Agila, Mendoza María, Ignacio Aguaded

La credibilidad publicitaria en la nueva esfera mediática: los universitarios y los medios

Advertising credibility in the new media sphere: university students and the media 
Arco iris en medios brasileños: percepciones sociales sobre una campaña publicitaria LGBTQIA+

Rainbow in Brazilian media: social perceptions of an LGBTQIA+ advertising campaign

Leonardo Ferreira Batista, José Geraldo de Araújo Ramalho Filho

224-239

Rituales y comunicación política: la toma de posesión de los presidentes autonómicos españoles

Rituals and political communication: the Spanish autonomous Presidents inauguration

Ricardo Domínguez García

240-258

RESEÑAS REVIEWS

Una mirada diferente para hacer periodismo

A different look for making journalism

Guillermo Paredes-Otero

259-261

Periodismo y tecnología: una simbiosis continua

Journalism and technology: a continuous symbiosis

Luisa Graciela Aramburú Moncada

262-265

Una propuesta metodológica para impartir la asignatura de redacción periodística

Journalism and technology: a continuous symbiosis

Noelia Ruiz-Alba

266-268 


\title{
Generación Z y consumo de información política: entre la televisión y los nuevos formatos mediáticos
}

\author{
Generation $Z$ and consumption of political information: between television \\ and new media formats
}

\author{
Ruth Gómez de Travesedo Rojas \\ Universidad de Málaga | Calle de León Tolstoi, s/n, Málaga 29010 | España \\ | https://orcid.org/0000-0003-4452-5928 | ruthgtr@uma.es \\ Marta Gil Ramírez \\ Universidad de Málaga | Calle de León Tolstoi, s/n, Málaga 29010 | España \\ | https://orcid.org/0000-0002-0577-5628 | martagr@uma.es
}

Fechas | Recepción: 29/05/2020 | Aceptación: 03/08/2020 | Publicación final: 15/10/2020

\begin{abstract}
Resumen
Los jóvenes de la Generación Z, definidos como la primera generación 100\% digital (Quintana, 2016; Vilanova y Ortega, 2017), suponen un nuevo reto para los políticos de todo el mundo. Acostumbrada al uso de medios convencionales para dirigirse a su audiencia, la esfera política ha tenido que replantear sus estrategias comunicativas para adaptarlas a una generación que apenas los consume, totalmente volcada en Internet y las redes sociales. En este contexto son numerosas las investigaciones que abordan el uso que los partidos políticos hacen de las redes sociales como instrumentos de comunicación hacia las audiencias más jóvenes; sin embargo, son prácticamente inexistentes aquellas que analizan dicho fenómeno desde la perspectiva contraria, la de los nativos digitales. El objetivo de la propuesta es analizar los medios de comunicación elegidos por los jóvenes de la generación Z para informarse sobre aspectos políticos. Se propone una investigación
\end{abstract}


exploratoria que emplea como instrumento metodológico el cuestionario estructurado, aplicado a estudiantes del grado de Publicidad y Relaciones Públicas de la Universidad de Málaga. Los resultados muestran cómo los jóvenes se debaten entre la televisión, Internet y las redes sociales entre los medios elegidos a la hora de informarse sobre asuntos de interés político de su entorno. En conclusión, la generación Z manifiestan un alto interés hacía la política, lo que contrasta con la percepción mayoritaria de no estar suficientemente informados al respecto. Esta contradicción invita a reflexionar sobre la idoneidad de las estrategias de comunicación que la política está implementando, especialmente en redes sociales.

Palabras clave: generación Z, comunicación política, jóvenes, redes sociales. instrument, applied to students of the degree of Advertising and Public Relations of the University of Malaga.The results show how young people debate themselves between television, the Internet and social networks among the media chosen to learn political issues. In conclusion, Generation Z shows a high interest in politics, which contrasts with the majority perception of not being sufficiently informed about it. This contradiction invites us to reflect on the adequacy of the communication strategies that politics are implementing, especially on social networks.

Keywords: generation Z, political communication, youth, social networks.

\section{INTRODUCCIÓN}

Es evidente que en la sociedad digital las estrategias de comunicación política se están adaptando a las posibilidades que ofrecen Internet y las redes sociales.

En el ámbito español, en los últimos años han proliferado los trabajos que abordan el desarrollo de la comunicación política en redes sociales y la interacción que en ellas se produce entre la esfera política y los usuarios de este tipo de plataformas digitales. La mayor parte de estos estudios, bien centrados en Twitter, donde la investigación es más prolija (Alonso, Marcos y Casero-Ripollés, 2016; Angulo, Estrella y López, 2018; Campos, 2017; Galán, 2017; GómezCalderón, Roses y Paniagua-Rojano, 2017; Jivkova, Requeijo y Padilla, 2017; López, Marcos y Casero-Ripollés, 2017; Marín, Simancas y Berzosa, 2019; Marcos, 2018; Miquel, Alonso y Marcos, 2017; Ruiz del Olmo y Bustos, 2016 y 2018; Suau-Gomila, 2020), Facebook (Abejón y Mayoral, 2017; Ballesteros y Díez, 2018; Fenoll y Cano, 2017; Muñiz, Dader, Téllez y Salazar, 2016) o YouTube (Berrocal, Gil y Campos, 2016; Berrocal, Martín y Gil, 2017; Gil, 2019a y 2019b; Gil y Gómez de Travesedo, 2020; Gil, Gómez de Travesedo y Almansa, 2019), apuntan en la misma dirección: a pesar de que los políticos de nuestro país valoran la importancia de estar en las redes sociales el uso que hacen de las mismas no es el adecuado.

Esto podría explicar el hecho de que, aunque grandes consumidores de medios online y redes sociales, el $51 \%$ de los jóvenes afirmen que para informarse sobre política recurren a la televisión, un $17 \%$ a informativos online y solo un $10 \%$ se informa de estos temas a través de redes sociales (Pin, 2018). ¿Responde esta contradicción entre los medios digitales que consumen masivamente los jóvenes y su elección de medios tradicionales para informarse sobre los asuntos políticos a la dificultad de los partidos y representantes para articular estrategias comunicativas en redes sociales que despierten el interés de los jóvenes? 
También fuera de nuestras fronteras son múltiples los trabajos que acercan el debate de la comunicación política a los contextos de las redes sociales desde distintas perspectivas: Aparaschivei (2011); Enli y Skogerbø (2013); Frame y Brachotte (2015); Vaccari, Valeriani, Barberá, Bonneau, Jost, Nagler, y Tucker (2013), entre otros; evidenciando el creciente interés de la esfera política en el nuevo escenario digital representado por los social networks.

Distintos estudios ponen de manifiesto el empeño que la clase política realiza por acercarse al público más joven, a través de su incursión en las redes sociales más populares entre este colectivo como Instagram (Cartes, 2018; Quevedo y Portalés, 2017; Lalancette y Raynauld, 2019; Liebhart y Bernhardt, 2017; Marcos, 2018; Názaro, Crozzoli y Álvarez-Nobel, 2019; Selva y Caro, 2017; Verón y Pallarés, 2018) o Snapchat (Gutiérrez y Peytibi, 2016; Morales y Romo, 2018), lo que refleja el interés creciente desde el ámbito político por el voto juvenil. Acercarse a los potenciales jóvenes votantes obliga a los políticos a reinventar sus estrategias comunicativas y migrarlas desde redes sociales como Facebook o Twitter a otras donde los nativos digitales representan actualmente el público mayoritario.

Aunque son numerosos los trabajos que han examinado la comunicación política en los social networks, generalmente estos ponen el foco de atención en el aprovechamiento que la esfera política hace de estos espacios digitales y, a pesar de que en ellos los jóvenes son usuarios mayoritarios, apenas existen investigaciones que aborden en qué medida el consumo de información política por parte de la nueva generación digital se ve condicionado por su asidua participación en estas plataformas.

En esta investigación se analiza, utilizando una encuesta por muestreo no probabilístico aplicada a una muestra de 139 estudiantes del grado de Publicidad y Relaciones Públicas de la Universidad de Málaga (UMA), el uso que los jóvenes de la llamada generación $\mathrm{Z}$ hacen de los medios de comunicación como fuente de información política.

Cabe destacar en este sentido, por su cercanía al objeto de estudio de la presente propuesta, la aportación de Villanueva, Aguilar y Sánchez (2017). Centrada en el ámbito local de Cataluña, esta investigación analiza el uso que los jóvenes del último curso de enseñanza obligatoria hacen de las redes sociales como plataformas de información y discusión política, relacionando la utilización de los medios, el nivel de uso de las redes sociales y el nivel de interés político de los adolescentes. Entre sus resultados destaca el hecho de que los jóvenes "para informarse sobre política siguen recurriendo a las mismas fuentes de información (diarios, radio y televisión), pese a ser usuarios intensivos de las redes sociales" (p. 80).

\section{REVISIÓN TEÓRICA Y PLANTEAMIENTO DEL PROBLEMA}

La televisión se ha configurado como el medio preferido para informarse sobre política en nuestro país, especialmente en época de elecciones. El Barómetro de diciembre 2019 del Centro de Investigaciones Sociológicas (CIS) refleja que el 56,5\% de los encuestados eligieron este medio como primera opción durante la campaña electoral de las elecciones generales del 10 de noviembre de 2019. 
Sin embargo, es una realidad que el consumo mediático de la población española está cambiando. Los datos facilitados por la Asociación para la Investigación de Medios de Comunicación (AIMC) en su Marco General de los Medios en España 2020 revelan como en los últimos diez años ha descendido la audiencia de los medios convencionales y ha aumentado la de Internet, especialmente entre los rangos de edad de la población más joven, siendo el porcentaje de penetración de Internet el más alto (96,5\%) en la franja de 20 a 24 años.

No solo se aprecia un cambio en el trasvase de audiencia de un medio a otro, sino que incluso la forma de acceder a ellos se ha modificado. Los dispositivos móviles han permitido a la audiencia consumir contenidos en cualquier lugar y a cualquier hora (Gil y Gómez de Travesedo, 2018). Lejos quedan ya los horarios sujetos a las parrillas televisivas o el consumo de televisión en el hogar; más de un $60 \%$ de la población accede a Internet en la calle o en los medios de transporte y un $94,2 \%$ lo hace a través de sus smartphones (AIMC, 2019).

La televisión tradicional ha sido sustituida por la televisión en streaming a través de plataformas como Neflix, HBO o Amazon Prime Video, donde el espectador no es solo un receptor pasivo de la oferta que le viene impuesta, sino que tiene la posibilidad de elegir qué ver, cuándo y, gracias a los dispositivos móviles, también dónde.

Por otra parte, la juventud no concibe su vida sin los social media, hasta el punto de que "el acceso a las nuevas tecnologías de la información y la comunicación, y todas sus infinitas posibilidades forma parte de su vida cotidiana" (García y Del Hoyo, 2013, p. 113). Los jóvenes son los usuarios que mayor número de redes sociales utilizan. Sus preferidas son YouTube, Instagram, Twitter, Snapchat o Tik Tok, según se desprende del informe elaborado por IAB Spain Estudio Anual de Redes Sociales 2019 y del estudio Digital 2020 Global Digital Overview realizado por We are Social y Hootsuite.

Los medios sociales están presentes en la vida diaria de los jóvenes. Son, sin duda, su espacio natural, desde el que realizan actividades muy diversas como compartir sus fotos, aficiones o vídeos curiosos, así como establecer conversaciones con su comunidad más cercana e informarse de la actualidad informativa a través, por ejemplo, de los perfiles que los medios digitales - tanto las versiones online de los tradicionales como los nativos - han abierto en estos espacios, precisamente para llegar a estos públicos mucho más reticentes a leer información en un medio convencional. En este contexto, adquieren un papel fundamental los dispositivos móviles, cuyo uso es creciente en todos los segmentos de edad, pero especialmente entre los más jóvenes. (Yuste, 2015, p. 179)

Los usuarios españoles destinan a las redes sociales una media de 55 minutos al día, siendo los más jóvenes los que dedican mayor tiempo a ellas, 50 minutos de media (IAB Spain, 2019).

En gran medida, los jóvenes de la llamada generación Z, son los responsables de estos cambios que se están generando en el consumo mediático. En este contexto los representantes políticos de nuestro país, si quieren llegar a sus futuros votantes, saben que tienen que adaptar sus estrategias de comunicación política. Así, los jóvenes nativos digitales se configuran como el nuevo reto de los políticos del siglo XXI. 


\subsection{La generación $Z$ y su relación con la política}

Aunque los distintos autores parecen no ponerse de acuerdo en la fecha exacta de nacimiento de los jóvenes pertenecientes a la generación $Z$, la mayoría de las taxonomías de las generaciones coinciden en señalar que se trataría de los nacidos entre 1994 y 2010. Jóvenes que actualmente tienen hasta 26 años y que han nacido ya en plena era digital. En nuestro país suponen casi 8 millones de personas y más de un 25\% de la población (Vilanova y Ortega, 2017).

A diferencia de la generación anterior, los millennials - que se debatían entre lo digital y lo analógico-, esta nueva generación de postmillennials se desenvuelve por completo en un contexto totalmente digital y no ha conocido un mundo sin Internet y sin redes sociales. "Son la primera generación 100\% digital” (Quintana, 2016, p. 128).

Se trata de "una generación que no necesita aprender las nuevas funcionalidades tecnológicas, ya que el mundo hiperconectado está presente en lo que constituye su propia naturaleza" (Marcelino, 2015, p. 51) y que "han aprendido antes a manejar el interface tecnológico que a leer y escribir" (Gabelas y Marta, 2011, p. 11).

Se conocen como generación $Z$ porque suceden a la generación Y (los millennials), aunque, desde el ámbito académico, reciben distintas denominaciones: entre otras, nativos digitales (Prensky, 2001), generación V, por virtual; generación C, por comunidad o contenido; generación de Internet; generación Google (Fernández y Fernández, 2016); Screenager o adolescentes de la pantalla (Gama, 2003); generación Net (Oblinger y Oblinger, 2005) o iGeneración, por la interactividad (Marta, Martínez y Sánchez, 2013). Sin embargo, con independencia de los distintos términos empleados para designarlos, se diferencian, entre otros aspectos, de las generaciones anteriores en el consumo de medios de comunicación y en su relación con la política.

Los jóvenes españoles de entre 20 y 24 años son poco fieles a los medios de comunicación tradicionales. Consumen información o entretenimiento cuando y donde quieren a través de sus dispositivos móviles. Aunque la televisión sigue siendo el medio más consumido entre este colectivo, ha ido perdiendo su liderazgo frente a Internet y las redes sociales. "Las redes están presentes en su día a día, desempeñando un papel clave en su vida cotidiana e influyendo notablemente en sus relaciones sociales, el consumo o el aprendizaje" (Marín y Simancas, 2019, p. 92). Además, el consumo televisivo que hacen se aleja del convencional y, habitualmente, no utilizan el medio como soporte de información sino de entretenimiento (López, Gómez y Medina, 2019).

Internet les ha permitido transformar el consumo de productos audiovisuales, proporcionándoles una "oferta variada, de acceso ilimitado, inmediato y ubicuo" que les posibilita elegir aquellos contenidos que desean visualizar cuando y donde deseen (García, Tur y Pastor, 2018, p. 31).

Definidos en base a las cuatro íes: Internet, irreverencia, inmediatez e incertidumbre (Vilanova y Ortega, 2017), la desconfianza política es también una de sus características principales. 
La incertidumbre que los acompaña viene marcada por el mundo líquido (Bauman, 2015) en el que les ha tocado vivir y la crisis económica de 2008 que marcó su infancia. Saben que estudiar no les asegura un buen trabajo y temen por su futuro. Pero no se conforman, y son irreverentes, cuestionándolo todo (Vilanova y Ortega, 2017). Además, gracias a Internet pueden hacerlo porque tienen acceso a infinitas fuentes de información.

Sumidos en la sociedad digital que cambia y avanza a un ritmo vertiginoso, consideran que la política es inmovilista y que España se encuentra en una situación de estancamiento en este sentido, a lo que se suma el desgaste generalizado de la credibilidad del sistema político del país (Vilanova y Ortega, 2017); de forma que se caracterizan por su desafección política. La desconfianza y distanciamiento hacia la clase política es evidente, como indica Echevarría (2013, p. 42) al señalar que "los jóvenes tienen una información mínima de los acontecimientos y actores políticos y que por lo tanto manifiestan un alto grado de apatía y despolitización".

Se trata de una generación que en su adolescencia vivió el Movimiento 15-M y la ruptura del bipartidismo político en nuestro país y, aunque interesada en el ámbito político, parece encontrar dificultades a la hora de identificarse con un partido en concreto. En este contexto no es de extrañar que el comportamiento político de estos jóvenes se caracterice por un voto volátil que tiende a afianzar los resultados de partidos de tercera generación como Podemos, Ciudadanos o Vox, renegando del bipartidismo tradicional. Es un colectivo altamente comprometido con el activismo en materias como el feminismo o el ecologismo (Suárez y Zuil, 2019) sobre las que los llamados partidos emergentes suelen ofrecer propuestas más contundentes.

\subsection{Nuevas estrategias de comunicación política: de Twitter a Instagram y Snapchat}

Los primeros postmillennials, aquellos nacidos entre los años 1995 y 1997, se estrenaron en unas elecciones generales en 2015; aunque la mayor parte lo hicieron el pasado abril -y nuevamente en noviembre- de 2019 y un mes más tarde, en mayo, pudieron expresar su voto en las municipales, autonómicas y europeas. Esta primera cita con las urnas obligó a las distintas formaciones políticas españolas a plantearse cómo atraer ese voto imprevisible de los jóvenes.

Los políticos han tenido que buscar nuevas estrategias para poder llegar e impactar en estos jóvenes que son más difíciles de influenciar por las formaciones con sus métodos tradicionales.

Es evidente que como sostienen Túñez y Sixto $(2011$, p. 1) en este contexto "[...] las redes sociales se erigen como un recurso y canal adecuado para la implementación del marketing político 2.0, creando un escenario donde es posible la interacción con los usuarios". Las redes sociales se configuran como los nuevos medios de las estrategias de comunicación política, dejando a un lado los medios tradicionales que pierden progresivamente eficacia para acercarse a los votantes más jóvenes. Se convierten en puntos de encuentro y discusión, donde los candidatos necesitan estar para interaccionar con los ciudadanos, teniendo presente que los individuos tienen en parte el control de lo que quieren ver o escuchar, por lo que deben afinar para conseguir generar la impresión de un mensaje individualizado si no quieren que este pase desapercibido o sea ignorado (Ariza, 2016; Gil y Gómez de Travesedo, 2020). 
Resulta por tanto lógico que las estrategias comunicativas de la esfera política de nuestro país hayan cambiado y se hayan adaptado al actual contexto de la sociedad digital. Como sostiene Comorera $(2017$, p. 9) "hoy en día no se puede entender la sociedad, sus modos de vida y sus problemas sin tener un ojo puesto en las redes sociales". Y los políticos son conscientes de que, para dirigirse a la sociedad, y especialmente a los jóvenes de la generación $Z$, deben cambiar sus flujos comunicativos.

Verón y Pallarés (2018) destacan como Instagram es empleada como herramienta de marketing político para trabajar la marca personal de los candidatos y trasmitir una imagen más personal, natural y cercana a la población. Respecto a Snapchat, dicha red social cuenta con la ventaja de acercar la figura del representante político a los jóvenes, contribuyendo a la cercanía y humanización del mismo, permitiendo incluso un toque de humor (Aduriz, 2016), por lo que se ha convertido en una red social ya empleada por políticos como Bernie Sanders o Hillary Clinton.

La relación de los jóvenes con Internet y las redes sociales queda también evidenciada en el estudio de la agencia M\&C Saatchi, que afirma que el $66 \%$ de los jóvenes españoles votaría en las elecciones si lo pudiese hacer por Internet y el $44 \%$ asume que sería más probable que votasen si se lo recordasen con un mensaje de texto (Nombelas, 2018). Los políticos, conocedores de esta situación, incluyen las redes sociales en sus estrategias comunicativas con el objeto de acercarse a una generación que parece que se les escapa.

Internet y las redes sociales se han convertido para muchos de ellos en un nuevo ámbito político, donde intercambiar opiniones, influir y construir sus propias subjetividades políticas, influyendo decisivamente en sus hábitos políticos más allá de la red. No se trata, pues, tanto de un desinterés por la política y los asuntos públicos, sino de un rechazo a unas prácticas y unos actores políticos que consideran ineficaces y alejados de sus intereses y de su capacidad de interlocución y la búsqueda de canales alternativos de participación política. (Lobera y Rubio, 2015, p. 145)

\subsection{Objetivos y metodología}

El objetivo de la presente investigación es analizar qué medios de comunicación eligen los jóvenes de la generación Z para informarse sobre los aspectos políticos de su país. Se pretende indagar sobre sus medios preferidos, no solo a la hora de recibir información en general sino, más concretamente, cuando dicha información está relacionada con asuntos relativos al contexto y circunstancias políticas de su entorno.

De este modo podremos comprobar si los medios que consumen habitualmente son los mismos que emplean a la hora de buscar información política, con el objeto de determinar si las estrategias de comunicación política actuales se están dirigiendo a los soportes adecuados.

La investigación, de carácter exploratorio, nos va a permitir identificar posibles pautas de consumo de información política por parte de los jóvenes de la generación $\mathrm{Z}$ así como relaciones potenciales entre los medios más consumidos por ellos a nivel general y aquellos a los que más recurren en la búsqueda de información política. El estudio se aplica a una muestra de 139 
estudiantes del grado de Publicidad y Relaciones Públicas de la Universidad de Málaga, empleando como instrumento de recogida de datos la encuesta por muestreo no probabilístico.

La elección del alumnado de dicha rama del conocimiento responde al interés por trabajar con grupos cercanos al consumo de información, inclinación que se les presupone a estos estudiantes que han optado por una formación vinculada con las múltiples vertientes que engloba el ámbito de la comunicación a través de un amplio corpus de asignaturas que, sobre el mismo, se imparten en dicho grado (Comunicación Política, Comunicación y Sociedad, Comunicación Institucional, Comunicación Cultural, etc.).

Se ha descartado la aplicación del estudio a estudiantes de la rama de Periodismo ante el riesgo que pudiera existir de que sus respuestas se vean influenciadas por sus conocimientos profesionales sobre los mass media. Con la elección de los estudiantes del grado de Publicidad y Relaciones Públicas nos aseguramos de un lado el interés de los individuos por el acceso a la información y, de otro, evitamos que sus respuestas puedan ofrecer un sesgo de características impostadas, de modo que adopten las respuestas que desde su contexto como futuros profesionales de la información considerarían más apropiadas.

En la encuesta participaron un total de 143 estudiantes. Cuatro de ellos fueron descartados por no cumplir el requisito de edad necesario para su pertenencia a la generación Z; por lo que la muestra quedó finalmente delimitada por 139 estudiantes con edades comprendidas entre los 18 y los 26 años.

El cuestionario, de carácter anónimo, recoge diversas preguntas (además de variables sociodemográficas) sobre el consumo mediático de información política, algunas de opción múltiple. Entre otros aspectos se interroga a los jóvenes sobre qué medios de comunicación consumen, cuáles consideran que gozan de mayor credibilidad y cuáles eligen a la hora de informarse sobre temas políticos, así como a los que recurrieron para informarse en las últimas elecciones generales. También se incluye una pregunta en la que se les pide que valoren según una escala psicométrica de Likert de 5 puntos la afirmación: "Internet y las redes sociales favorecen la proliferación de fake news".

\section{RESULTADOS}

Los datos obtenidos reflejan una evidente contradicción entre el uso que los jóvenes realizan de los medios como fuente de información y la credibilidad que le otorgan a los mismos. El medio que obtiene mayores niveles de credibilidad, la prensa, aparece sin embargo entre los menos empleados por los estudiantes encuestados para mantenerse informados. Para estos fines, los miembros de la generación $Z$ que componen el estudio optan principalmente por las redes sociales $(31,9 \%)$, Internet $(29,7 \%)$ y la televisión $(26,5 \%)$. 


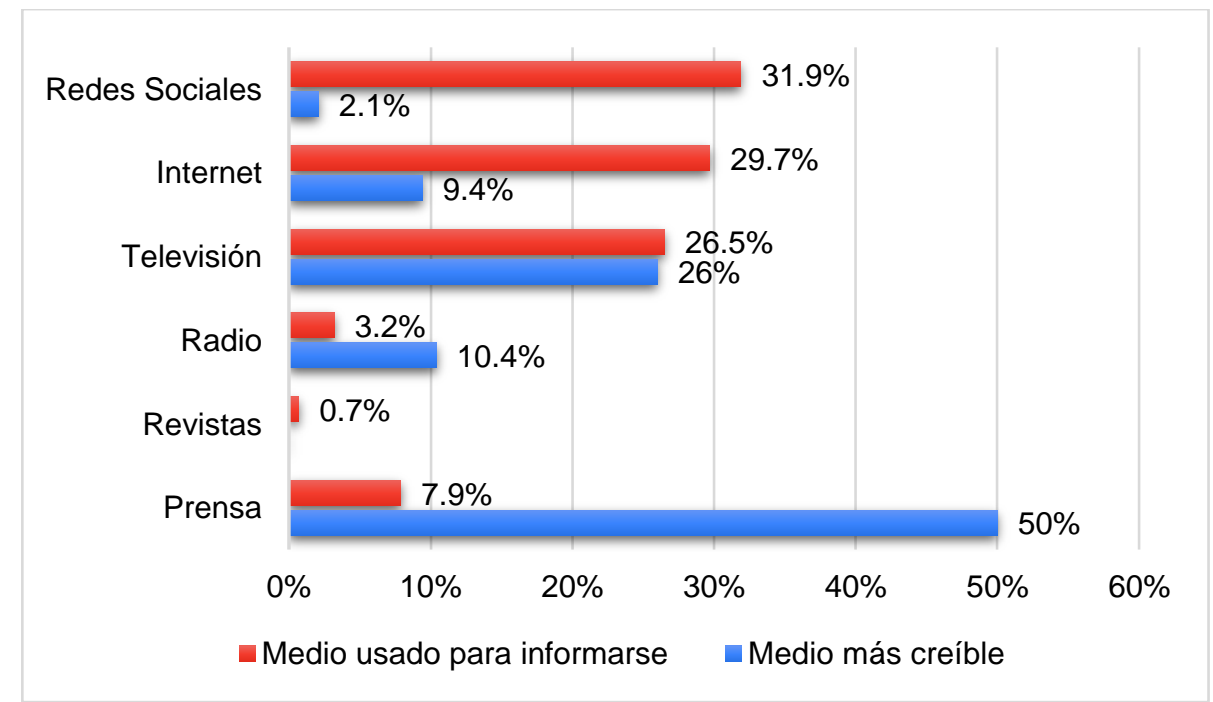

Gráfico 1. Uso como fuente informativa y credibilidad de los medios por parte de la generación Z. Fuente: elaboración propia.

Se observa una proporción inversa en el uso que los jóvenes hacen de las redes sociales, Internet y la prensa, y la credibilidad que le conceden a estos medios. Esta proporción es más equilibrada con relación a la televisión. La radio obtiene unos índices de credibilidad superiores a Internet, pero decae considerablemente como fuente informativa entre la juventud encuestada. Las revistas, por su parte resultan un medio prácticamente inexistente entre el colectivo examinado.

Nuevamente los datos resultan contradictorios al interrogar a los jóvenes sobre cómo de acuerdo están con la afirmación de que Internet y las redes sociales facilitan la proliferación de fake news. Pese al bajo índice de estudiantes que otorgaba credibilidad a estos medios, ante la afirmación propuesta, es superior el porcentaje de alumnos que muestra su desacuerdo (41,7\%), al que está bastante o muy de acuerdo $(38,5 \%)$ con el hecho de que en las redes sociales abunden las noticias falsas.

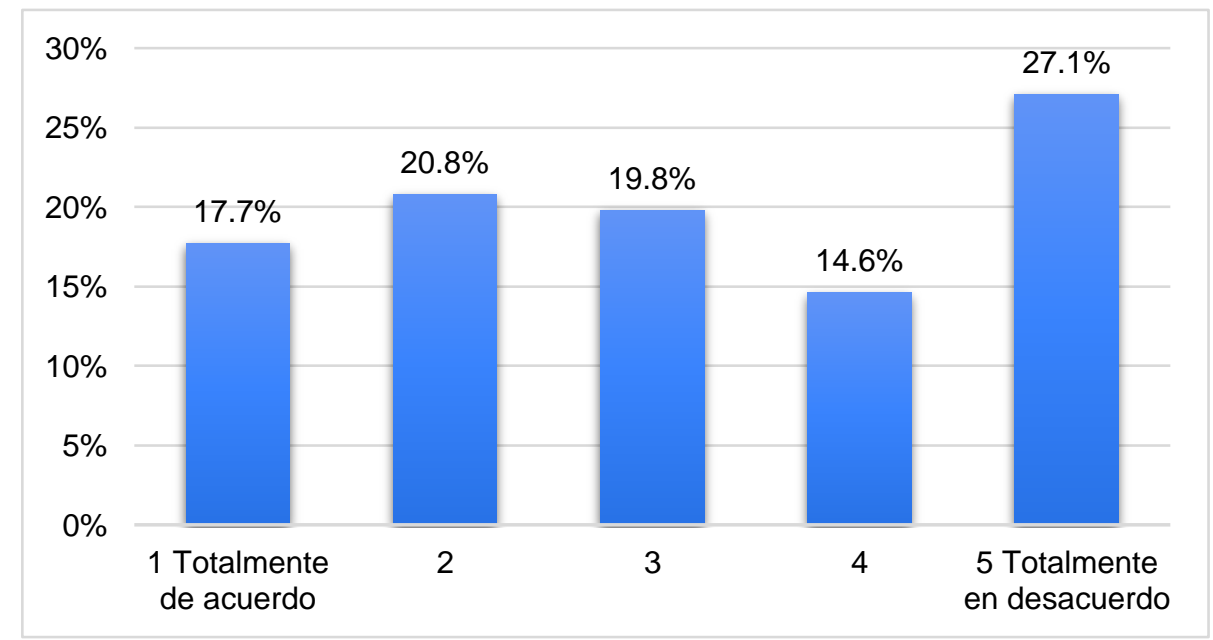

Gráfico 2. ¿Cómo de acuerdo estás con la afirmación de que "las redes sociales e Internet favorecen la proliferación de fake news"?

Fuente: elaboración propia. 
En general, los alumnos que participan del estudio son activos en el consumo informativo, siendo prácticamente unánime la práctica de contrastar la información $(90,7 \%)$. Si bien, un $46,4 \%$ de quiénes la contrastan lo hace de forma habitual frente a un $44,3 \%$ que recurre a segundas fuentes solo en determinadas ocasiones.

En cuanto a la relación de los jóvenes con la política, más de la mitad de los encuestados (58,3\%) se define con interés hacia la misma y un $55,6 \%$ afirma seguir el perfil de algún político o formación en redes sociales. Sin embargo, pese a este interés manifiesto, son mayoritarios los jóvenes de la generación $Z$ que consideran no estar suficientemente informados sobre los asuntos políticos de su país $(62,5 \%)$.

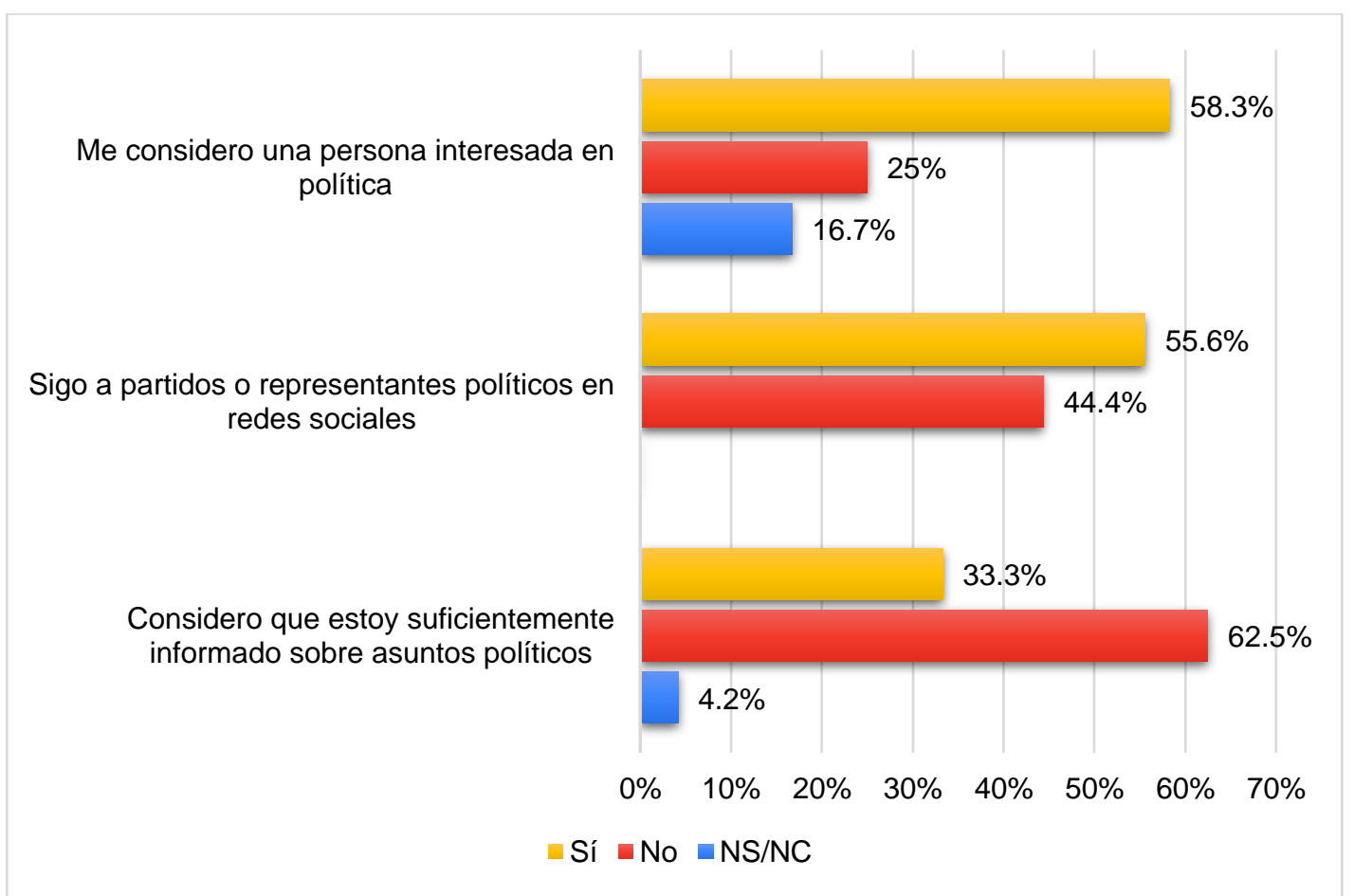

\section{Gráfico 3. Interés y nivel de información sobre asuntos políticos de la generación Z} Fuente: elaboración propia.

Con relación al medio preferido para informarse sobre política, los datos resultan similares a los que se desprenden del uso que los jóvenes realizan de los medios de comunicación como fuente de información genérica. Los alumnos que participan del estudio acceden a contenido de índole política principalmente mediante Internet $(33,3 \%)$, televisión $(24 \%)$ y redes sociales $(22,9 \%)$, siendo minoritario para estos fines el recurso a la prensa $(16,7 \%)$ y prácticamente marginal en relación con la radio $(3,1 \%)$. 


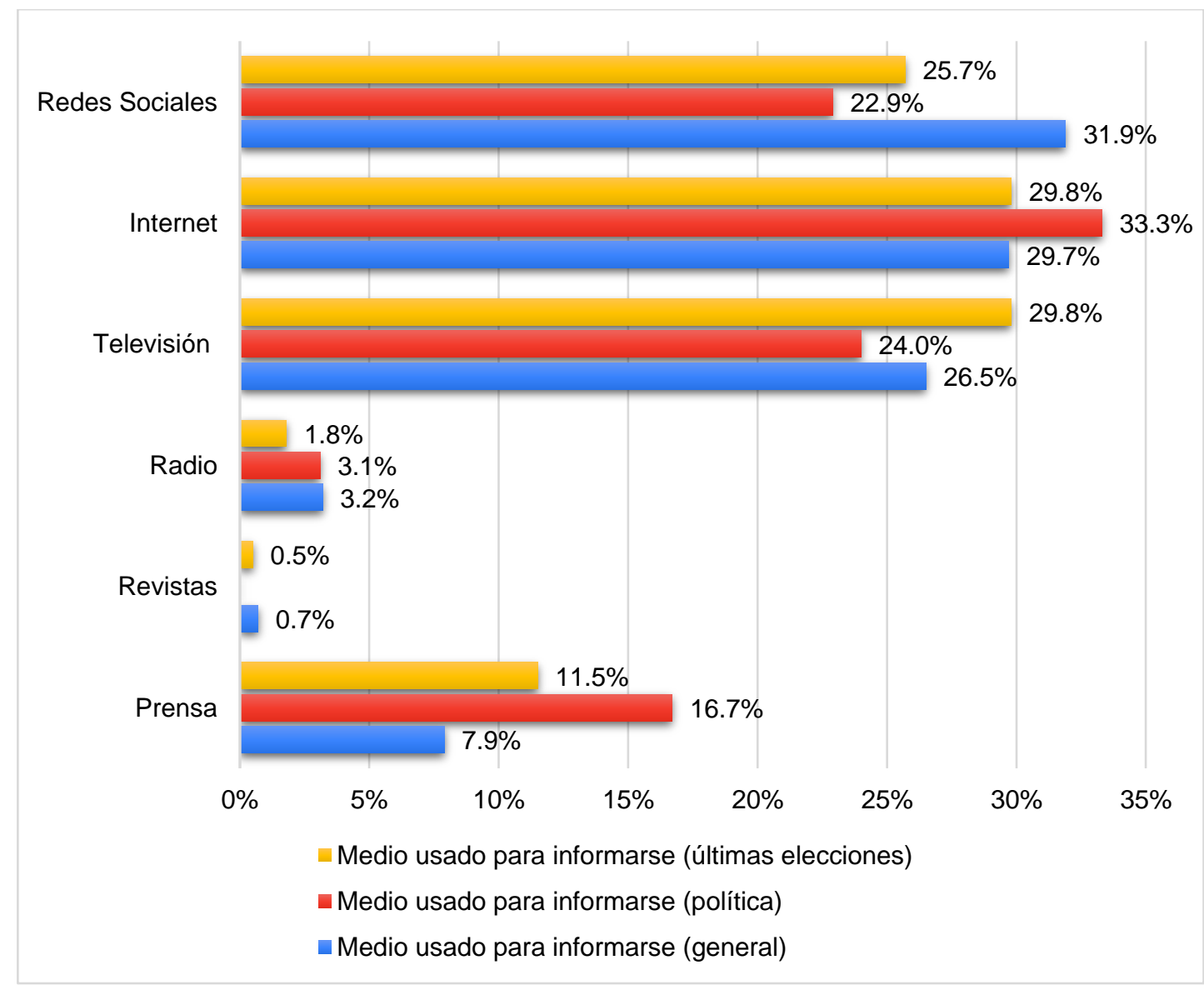

Gráfico 4. Medios empleados por la generación Z para informarse sobre política Fuente: elaboración propia.

Al cuestionarles sobre los medios de comunicación que emplearon para informarse en las últimas elecciones generales se repite la tendencia detectada hasta el momento: Internet y la televisión reflejaron porcentajes idénticos, con casi un 30\%, seguidas de cerca por las redes sociales $(25,7 \%)$ y a mayor distancia prensa $(11,5 \%)$ y radio $(1,8 \%)$. Se ratifica, por tanto, que estos tres medios (televisión, Internet y redes sociales) son los preferidos por los jóvenes a la hora de recibir información política.

Precisamente es la combinación de los tres medios que se evidencian como los más empleados por los jóvenes, tanto a nivel genérico como para informarse de aspectos políticos, la que presenta una mayor frecuencia de repetición $(20,8 \%)$ como fuente de información consultada durante las últimas elecciones generales. El $14,6 \%$ de los encuestados acudieron únicamente a Internet para obtener información electoral y un $11,5 \%$ se informó de forma exclusiva a través de la televisión. Por otra parte, prensa, revistas y radio no son empleados por los jóvenes como medios únicos de información política en el contexto de unos comicios, y las redes sociales solo lo son en un escaso $3,1 \%$ de los casos. 
Tabla 1

Medios elegidos por la generación Z para informarse en las últimas elecciones generales

\begin{tabular}{ll}
\hline \multicolumn{1}{l}{ Medios elegidos para informarse en las últimas elecciones generales } & $\%$ alumnado \\
\hline De forma aislada & 11,5 \\
\hline Televisión & 14,6 \\
\hline Internet & 3,1 \\
\hline Redes sociales & \\
\hline De forma combinada & 2,1 \\
\hline Prensa, redes sociales & 7,3 \\
\hline Televisión, Internet & 7,3 \\
\hline Televisión, redes sociales & 3,1 \\
\hline Prensa, televisión & 7,3 \\
\hline Internet, redes sociales & 1,0 \\
\hline Prensa, Internet & 4,2 \\
\hline Prensa, televisión, redes sociales & 20,8 \\
\hline Televisión, Internet, redes sociales & 3,1 \\
\hline Prensa, televisión, Internet & 1,0 \\
\hline Prensa, Internet, redes sociales & 3,1 \\
\hline Prensa, televisión, radio, Internet, redes sociales & 7,3 \\
\hline Prensa, televisión, Internet, redes sociales & 1,0 \\
\hline Prensa, revistas, Internet, redes sociales & 1,0 \\
\hline No me informé & 1,0 \\
\hline Programa electoral & \\
\hline Funte: elaboracion propia. & \\
\hline
\end{tabular}

Fuente: elaboración propia.

\section{DISCUSIÓN Y CONCLUSIONES}

Los jóvenes de la generación $Z$ se definen como personas con interés en la política. Se informan sobre estos temas y siguen a formaciones o representantes políticos en redes sociales. Aspecto este que diverge de los resultados obtenidos por Villanueva, Aguilar y Sánchez (2017) que concluyen un escaso interés político de los adolescentes que centran la investigación, esto es, estudiantes catalanes del último curso de Educación Obligatoria. Si bien, las diferencias en los hallazgos de ambos estudios pueden estar relacionadas con la distinta horquilla de edades de quienes componen las muestras en ambos casos.

El interés que los jóvenes encuestados manifiestan por los asuntos políticos de su país contrasta con el hecho de que una amplia mayoría no se siente suficientemente informado al respecto. Esta contradicción invita a reflexionar sobre la idoneidad de las estrategias de comunicación de la política actual y las vías que la esfera política española elige para trasladar sus mensajes a los jóvenes votantes.

Centrándonos en el consumo mediático, los jóvenes recurren mayoritariamente, como indican los estudios precedentes (García y Del Hoyo, 2013; Marín y Simancas, 2019; Villanueva, Aguilar y Sánchez, 2017; Yuste, 2015), a Internet y las redes sociales, aunque curiosamente otorgan a la prensa los niveles más altos de credibilidad, situándola como el medio más confiable, seguido 
de la televisión. Internet y las redes sociales, los medios que más consumen, no son considerados como los más verosímiles, aunque no están de acuerdo en que favorezcan la aparición de noticias falsas. Quizá porque consideran que este tipo de informaciones son comunes a todos los medios sin distinción, aspecto este que podría ser analizado con mayor detenimiento y profundidad en futuras investigaciones. El dato positivo, al margen del tipo de medio que empleen para informarse o el grado de credibilidad que le otorguen, lo encontramos en el elevado índice de estudiantes que recurren a distintas fuentes de información para contrastar la fiabilidad de la misma.

Aspecto, el del contraste informativo, que también queda evidenciado cuando la mayoría de los encuestados afirma haber recurrido a varios medios a la hora de informarse en las últimas elecciones generales, descartando la utilización de una única fuente informativa. Los resultados evidencian que los jóvenes son activos en la selección de las fuentes de información política, acudiendo a distintos medios y combinando medios tradicionales con nuevos medios $y$ plataformas digitales.

No podemos perder de vista que los encuestados son estudiantes de comunicación, por lo que la cifra tan alta de alumnos que verifican la información que reciben podría tener su lectura en la predisposición a dicha actividad que se les presupone como futuros profesionales del sector.

Como hemos señalado anteriormente, aunque otorgan gran credibilidad a la prensa escrita, para informarse sobre actualidad política prefieren hacerlo a través de la televisión, Internet o redes sociales, lo que denota la importancia que para esta generación tienen los contenidos audiovisuales y multimedia. Ese hecho evidencia un cambio de tendencia respecto de generaciones precedentes para las que la prensa y la radio eran consideradas medios de calidad y a las que tradicionalmente se les ha otorgado un alto grado de confianza.

Hasta aquí las conclusiones guardan similitud con los hallazgos de Villanueva, Aguilar y Sánchez (2017) que apuntan a la televisión y las redes sociales como los medios preferidos por los adolescentes para mantenerse informados sobre asuntos políticos $y$, por tanto, a una combinación, como la que resulta de la presente propuesta, de medios tradicionales y nuevos medios como fuente de información política. Si bien, la comparación con dicho estudio está limitada por el hecho de que, en su lectura, los autores atienden de forma individual a cada una de las redes sociales que examinan.

En cualquier caso, la presente investigación se perfila como una primera aproximación al uso que los jóvenes de la generación Z realizan de los medios de comunicación y las redes sociales como fuente de información política, evidenciando la necesidad apremiante de que la esfera política redirija sus estrategias comunicativas a estas últimas si persigue el objetivo de seducir el voto juvenil.

Esta propuesta pone sobre la mesa la posibilidad de iniciar diversas líneas de investigación que puedan arrojar luz sobre los formatos televisivos seleccionados (debates, informativos...) o las redes sociales concretas (Twitter, Instagram, Facebook...) a las que recurren los jóvenes para informarse sobre actualidad política. Análisis desde una perspectiva cualitativa permitirían 
igualmente determinar el motivo por el que los jóvenes no recurren a sus medios preferidos (redes sociales e Internet) a la hora de informarse sobre política, evidenciando si este comportamiento responde a una actitud caprichosa de los propios jóvenes o a la mala gestión de las estrategias de comunicación política en dichos medios, en los que aún se carece, como apuntan investigaciones precedentes (Alonso, Marcos y Casero-Ripollés, 2016; Angulo, Jivkova, Requeijo y Padilla, 2017; Ballesteros y Diez, 2018; Gil, 2019a, 2019b; Gil y Gómez de Travesedo, 2020; Gil, Gómez de Travesedo y Almansa, 2019; Marín, Simancas y Berzosa, 2019; Miquel, Alonso y Marcos, 2017; Muñiz, Dader, Téllez y Salazar, 2016), de una verdadera interacción entre la clase política y los usuarios de estas plataformas digitales.

Por otra parte, hacer extensivos estudios de este tipo a una muestra mayor podría permitirnos comprobar si el comportamiento detectado en los alumnos encuestados se repite en el resto de los jóvenes de la generación $\mathrm{Z}$ o incluso advertir si el nivel de estudios o la rama de conocimiento influye en la elección del medio de comunicación a la hora de informarse sobre política, en cuyo caso la variable generacional quedaría en entredicho.

Nos encontramos ante una nueva generación que ha nacido y crecido en un entorno totalmente digital y que reclama una reformulación de las estrategias comunicativas de la esfera política en los nuevos medios ya que, como reflejan los resultados, los jóvenes de la generación Z siguen recurriendo a los legacy media para informarse sobre política, a pesar de que desarrollan gran parte de su día a día a través de las redes sociales.

\section{Referencias}

Abejón, P. y Mayoral, J. (2017). Persuasión a través de Facebook de los candidatos en las elecciones generales de 2016 en España. El profesional de la información (EPI), 26(5), 928-936. doi: 10.3145/epi.2017.sep.14

Aduriz, I. (8 de octubre de 2016). Snapchat, nueva arma política. Cambio 16. Recuperado de https://bit.ly/3d0RtqV

AIMC (2019). Marco general de los medios en España 2020. Recuperado de https://bit.ly/3ccHNbA

Alonso, L.; Marcos, S., \& Casero-Ripollés, A. (2016). Political leaders in (inter)action. Twitter as a strategic communication tool in electoral campaigns. Trípodos, (39), 71-90. Recuperado de https://bit.ly/2zw3m9G

Angulo, N. A.; Estrella, A. y López, M. (2018). La política en Twitter. Un estudio comparativo de las estrategias discursivas de los candidatos finalistas a la Presidencia de Ecuador en 2017. adComunica. Revista Científica de Estrategias, Tendencias e Innovación en Comunicación, (16), 25-44. doi: 10.6035/2174-0992.2018.16.3

Aparaschivei, P. A. (2011). The use of new media in electoral campaigns: Analysis on the use of blogs, Facebook, Twitter and YouTube in the 2009 Romanian presidential campaign. Journal of Media Research-Revista de Studii Media, 4(10), 39-60.

Ariza, A. (2016). Las estrategias comunicativas, en Twitter y Facebook, en la campaña electoral presidencial 2015. En V Congreso Internacional en Comunicación Política y Estrategias de Campaña. Asociación Latinoamericana de Investigadores en Campañas Electorales. Recuperado de https://bit.ly/2A9IZjY 
Ballesteros, C. A. y Díez, M. (2018). Tenemos que hablar. El Compromiso 2.0 en Facebook durante la cibercampaña española del 20D de 2015. Comunicación y sociedad, 31(1), 169-193. doi: 10.15581/003.31.1.169-193

Bauman, Z. (2015). Modernidad líquida. México: Fondo de cultura económica.

Berrocal, S., Gil, A. y Campos, E. (2016). El uso de YouTube en las elecciones al Parlamento Europeo 2014. El caso de España. Comunicación y hombre, (12), 57-72. doi: 10.32466/eufv-cyh.2016.12.189.5772

Berrocal, S., Martín, V. y Gil, A. (2017). Líderes políticos en YouTube información y politainment en las elecciones generales de 2016 (26J) en España. El profesional de la información (EPI), 26(5), 937946. doi: 10.3145/epi.2017.sep.15

Campos, E. (2017). Twitter y la comunicación política. El profesional de la información (EPI), 26(5), 785793. doi: 10.3145/epi.2017.sep.01

Cartes, M. J. (2018). El uso de Instagram por los partidos políticos catalanes durante el referéndum del 1O. Revista de la SEECI, (47), 17-36. doi: 10.15198/seeci.2018.47.17-36

CIS (2019). Barómetro de diciembre 2019. Postelectoral Elecciones Generales 2019. Recuperado de https://bit.ly/2ZHOiRd

Comorera, J. (2017). Prologo. En A. Chaves-Montero (edit.), Comunicación política y redes sociales, pp. 910. Sevilla: Ediciones Egregius.

Echevarría Victoria, M. (2013). ¿Apatía o desencuentro? Patrones de consumo y recepción de información política y gubernamental en jóvenes. Global Media Journal México, 8(15), 42-65. Recuperado de https://bit.ly/2B163jY

Enli, G. S., \& Skogerb $\varnothing$, E. (2013). Personalized campaigns in party-centred politics: Twitter and Facebook as arenas for political communication. Information, communication \& society, 16(5), 757-774. doi: 10.1080/1369118X.2013.782330

Fenoll, V. y Cano, L. (2017). Participación ciudadana en los perfiles de Facebook de los partidos españoles. Análisis de comentarios en la campaña electoral de 2015. Communication \& Society, 30(4), 131148. doi: 10.15581/003.30.3.131-148

Fernández, F. J. y Fernández, M. (2016). Los docentes de la Generación Z y sus competencias digitales. Comunicar, 24(46), 97-105. doi: 10.3916/C46-2016-10

Frame, A., \& Brachotte, G. (2015). Le tweet stratégique: Use of Twitter as a PR tool by French politicians. Public relations review, 41(2), 278-287. doi: 10.1016/j.pubrev.2014.11.005

Gabelas, J. A. y Marta, C. (2011). Adolescentes en la cultura digital. En E. Martínez Rodríguez y Marta Lazo, C. (Coords.), Jóvenes Interactivos. Nuevos modos de comunicarse (pp. 03- 15). La Coruña: Netbiblio

Galán, M. (2017). The 2016 Republican primary campaign on Twitter: Issues and ideological positioning for the profiles of Ben Carson, Ted Cruz, Marco Rubio, and Donald Trump. El profesional de la información (EPI), 26(5), 850-858. doi: 10.3145/epi.2017.sep.07

Gama, L. R. (2003). Jogos eletrônicos e violência: desvendando o imaginário dos screenagers. Revista da FAEEBA, 11(8), 437-446. Recuperado de https://bit.ly/3c5WPQI

García, A., Tur, V., y Pastor, Y. (2018). Consumo mediático de adolescentes y jóvenes. Noticias, contenidos audiovisuales y medición de audiencias. ICONO 14, Revista de comunicación y tecnologías emergentes, 16(1), 22-46. doi: 10.7195/ri14.v16i1.1101

García, M. y Del Hoyo, M (2013). Redes sociales, un medio para la movilización juvenil. Zer, 18(34), 111125. Recuperado de https://bit.ly/3c3PJf7 
Gil, M. (2019a). El uso de YouTube en el Procés catalán. Comunicación política a través de los social media: ¿prosumidores mediatizados? Estudios sobre el Mensaje Periodístico, 25(1), 213-234. doi: 10.5209/ESMP.63725

Gil, M. (2019b). ¿Participa la esfera política en YouTube? Producción y consumo de información en la campaña electoral catalana de 2017. Ámbitos. Revista Internacional de Comunicación, (44), 141161. doi: 10.12795/Ambitos.2019.i44.09

Gil, M. y Gómez de Travesedo, R. (2018). Uso del smartphone en el periodismo actual: ¿hacia un nuevo modelo mediático? Estudio de caso del contexto malagueño. Ámbito Revista Internacional de Comunicación, (41), 5-21. doi: 10.12795/Ambitos.2018.i41.01

Gil, M. y Gómez de Travesedo, R. (2020). Gestión de la política española en YouTube. Una asignatura pendiente. Observatorio (OBS*), 14(1), 22-44. doi: 10.15847/obsOBS14120201491

Gil, M., Gómez de Travesedo, R. y Almansa, A. (2019). Politainment y personalización política ¿De la televisión a YouTube? Revista Latina de Comunicación Social, (74), 1542-1564. doi: 10.4185/RLCS-2019-1398-81

Gómez-Calderón, B., Roses, S. y Paniagua-Rojano, F. J. (2017). La campaña en 140 caracteres. Empleo de Twitter por parte de los candidatos de los partidos políticos mayoritarios ante las elecciones generales de 2016 en España. El profesional de la información, 26(5), 816-823. doi: oi.org/10.3145/epi.2017.sep.04

Gutiérrez, A. y Peytibi, X. (2016). Snapchat en política. Transformando la comunicación social. Barcelona: LuzAzul ediciones.

IAB Spain (2018). Estudio Anual de Redes Sociales 2019. Recuperado de https://bit.ly/3d8raPv

Jivkova, D., Requeijo, P. y Padilla, G. (2017). Usos y tendencias de Twitter en la campaña a elecciones generales españolas del 20D de 2015: hashtags que fueron trending topic. El profesional de la información (EPI), 26(5), 824-837. doi: 10.3145/epi.2017.sep.05

Lalancette, M., \& Raynauld, V. (2019). The power of political image: Justin Trudeau, Instagram, and celebrity politics. American Behavioral Scientist, 63(7), 888-924. doi: $10.1177 / 0002764217744838$

Liebhart, K., \& Bernhardt, P. (2017). Political Storytelling on Instagram: Key Aspects of Alexander Van der Bellen's Successful 2016 Presidential Election Campaign. Media and Communication, 5(4), 15-25. doi: 10.17645/mac.v5i4.1062

Lobera, J. y Rubio, R. (2015). Nativos digitales: ¿hacia una nueva participación política? Revista de Estudios de Juventud, (108), 145-160. Recuperado de https://bit.ly/3c2md9K

López, N., Gómez, L. y Medina, E. (2019). Los formatos de televisión más consumidos por los jóvenes: telerrealidad y empoderamiento de la audiencia. Ámbitos. Revista Internacional de Comunicación, (46), 10-27. doi: 10.12795/Ambitos.2019.i46.02

López, A., Marcos, S., \& Casero-Ripollés, A. (2017). What do politicians do on Twitter? Functions and communication strategies in the Spanish electoral campaign of 2016. El profesional de la información (EPI), 26(5), 795-804. doi: 10.3145/epi.2017.sep.02

Marcelino, G. V. (2015). Migración de los jóvenes españoles en redes sociales, de Tuenti a Facebook y de Facebook a Instagram. La segunda migración. ICONO 14, Revista de comunicación y tecnologías emergentes, 13(2), 48-72. doi: 10.7195/ri14.v13i2.821 
Marcos, S. (2018). Las redes sociales como herramienta de la comunicación política. Usos políticos y ciudadanos de Twitter e Instagram (tesis doctoral). Universitat Jaume I. Recuperado de https://bit.ly/2M408Lv

Marín, P. P., Simancas, E. y Berzosa, A. (2019). Uso e influencia de Twitter en la comunicación política: el caso del Partido Popular y Podemos en las elecciones generales de 2016. Cuadernos.Info, (45), 129-144. doi: $10.7764 /$ cdi.45.1595

Marín, P. P., y Simancas, E. (2019). Los universitarios millennials: uso de redes sociales y relación con las marcas. Ámbitos. Revista Internacional de Comunicación, 46, 92-107. doi: 10.12795/Ambitos.2019.i46.06

Marta, C., Martínez, E. y Sánchez, L. (2013). La «i-Generación» y su interacción en las redes sociales. Análisis de Coca-Cola en Tuenti. Comunicar, 20(40), 41-48. doi: 10.3916/C40-2013-02-04

Miquel, S., Alonso, L. y Marcos, S. (2017). Buscando la interacción. Partidos y candidatos en Twitter durante las elecciones generales de 2015. Prisma Social, (18), 34-54. Recuperado de https://bit.ly/2X67NRH

Morales, E. I. y Romo, A. M. (2018). Las redes sociales como medio de comunicación política. El uso del Snapchat. Revista STATUS, 2(3), 37-58. Recuperado de https://bit.ly/3d5DJel

Muñiz, C., Dader, J. L., Téllez, N. M. y Salazar, A. (2016). ¿Están los políticos políticamente comprometidos?: Análisis del compromiso político 2.0 desarrollado por los candidatos a través de Facebook. Cuadernos.Info, (39), 135-150. doi: 10.7764/cdi.39.970

Názaro, A., Crozzoli, F., y Álvarez-Nobell, A. (2019). Comunicación política digital en Instagram: los casos de Cristina Fernández de Kirchner y Mauricio Macri en Argentina. Revista Internacional de Relaciones Públicas, 9(18), 5-28. doi: 10.5783/RIRP-18-2019-02-05-28

Nombelas, C. G. (18 de julio de 2018). Generación Z: así son los post-millennials. Forbes. Recuperado de https://bit.ly/2XwZhtY

Oblinger, D., y Oblinger, J. (2005). Educar a la generación net. EU: Educase.

Pin, J. R. (8 de febrero de 2018). Postmillennials (Generación Z) y política. elEconomista.es. Recuperado de https://bit.ly/2yxvJDY

Prensky, M. (2001). Digital Natives, Digital Immigrants. NCB University Press.

Quevedo, R., y Portalés, M. (2017). Imagen y comunicación política en Instagram. Celebrificación de los candidatos a la presidencia del Gobierno. El profesional de la información (EPI), 26(5), 916-927. doi: 10.3145/epi.2017.sep.13

Quintana, Y. (2016). Generación Z: vuelve la preocupación por la transparencia online. Revista de Estudios de Juventud, (114), 127-142. Recuperado de https://bit.ly/2XrGikw

Ruiz Del Olmo, F. J. y Bustos, J. (2016). Del tweet a la fotografía, la evolución de la comunicación política en Twitter hacia la imagen. El caso del debate del estado de la nación en España (2015). Revista Latina de Comunicación Social, (71), 108-123. doi: 10.4185/RLCS-2016-1086

Ruiz del Olmo, F. J. y Bustos, J. (2018). Los retweets de los candidatos como parte de la comunicación política. Estudios sobre el Mensaje Periodístico, 24(2), 1703-1713. doi: 10.5209/ESMP.62242

Selva, D. y Caro, L. (2017). Uso de Instagram como medio de comunicación política por parte de los diputados españoles: la estrategia de humanización en la "vieja" y la "nueva" política. El profesional de la información (EPI), 26(5), 903-915. doi: 10.3145/epi.2017.sep.12

Suárez, C. y Zuil, M. (7 de abril de 2019). El voto imprevisible de la Generación Z. El Confidencial. Recuperado de https://bit.ly/36CWOCA 
Suau-Gomila, G. (2020). Microblogging electoral: la estrategia comunicativa de Podemos y Ciudadanos en Twitter en las campañas electorales del 20D y el 26J. Prisma social, (28), 103-126. Recuperado de https://bit.ly/2X24LOS

Túñez, M. y Sixto, J. (2011). Redes sociales, política y Compromiso 2.0: La comunicación de los diputados españoles en Facebook. Revista Latina de comunicación social, (66), 1-25. doi: 10.4185/RLCS-662011-930-210-234

Vaccari, C., Valeriani, A., Barberá, P., Bonneau, R., Jost, J. T., Nagler, J., \& Tucker, J. (2013). Social media and political communication. A survey of Twitter users during the 2013 Italian general election. Rivista italiana di scienza politica, 43(3), 381-410. doi: 10.1426/75245

Verón, J. J. y Pallarés, S. (2018). La imagen del político como estrategia electoral: el caso de Albert Rivera en Instagram. Mediatika. Cuadernos de Medios de Comunicación, (16), 195-217. Recuperado de https://bit.ly/3gu77wX

Vilanova, N., y Ortega, I. (2017). Generación Z: Todo lo que necesitas saber sobre los jóvenes que han dejado viejos a los millennials. Barcelona: Plataforma Editorial.

Villanueva, S., Aguilar, C. y Sánchez, L. (2017). Participación política, medios de comunicación y redes sociales en los adolescentes catalanes. International Journal of Sociology of Education, 6(1), 6184. doi: $10.17583 /$ rise.2017.2496

We are Social y Hootsuite (2019). Digital 2020 Global Digital Overview. Recuperado de https://bit.ly/2M1hMBz

Yin, R. K. (1989). Case Study Research: Design and Methods. London: Sage publications.

Yuste, B. (2015). Las nuevas formas de consumir información de los jóvenes. Revista de estudios de juventud, (108), 179-191. Recuperado de https://bit.ly/3d8DgrS 